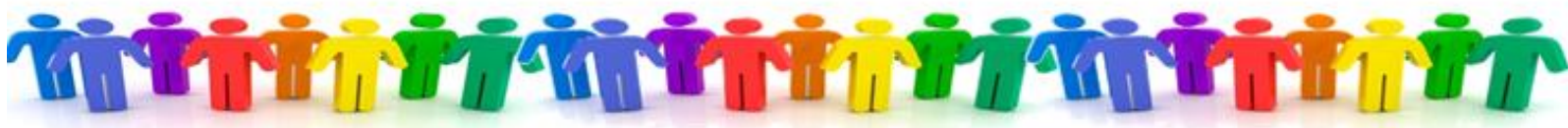

\section{Fear of Self-Annihilation and Existential Uncertainty as Predictors of Worldview Defense: Comparing Terror Management and Uncertainty Theories}

\author{
Mark Rubin \\ The University of Newcastle, Australia
}

Citation: Rubin, M. (2017). Fear of self-annihilation and existential uncertainty as predictors of worldview defence: Comparing terror management and uncertainty theories. Journal of Social Psychology. doi: $\underline{10.1080 / 00224545.2017 .1341375}$

\begin{abstract}
Terror management theory (TMT) proposes that thoughts of death trigger a concern about self-annihilation that motivates the defense of cultural worldviews. In contrast, uncertainty theorists propose that thoughts of death trigger feelings of uncertainty that motivate worldview defense. University students $(\mathrm{N}=414)$ completed measures of the chronic fear of self-annihilation and existential uncertainty as well as the need for closure. They then evaluated either a meaning threat stimulus or a control stimulus. Consistent with TMT, participants with a high fear of self-annihilation and a high need for closure showed the greatest dislike of the meaning threat stimulus, even after controlling for their existential uncertainty. Contrary to the uncertainty perspective, fear of existential uncertainty showed no significant effects.
\end{abstract}

Keywords: meaning threat; need for closure; terror management theory; uncertainty.

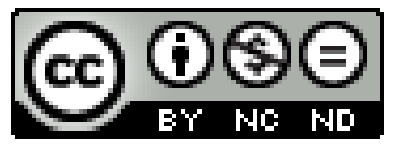

Copyright (C) The Author. OPEN ACCESS: This material is published under the terms of the Creative Commons Attribution-NonCommercial-NoDerivatives 4.0 International licence (CC BY-NC-ND 4.0; https://creativecommons.org/licenses/bync-nd/4.0/). This licence permits you to copy and redistribute this material in any medium or format for noncommerical purposes without remixing, transforming, or building on the material provided that proper attribution to the authors is given.

This self-archived article is provided for non-commercial and scholarly purposes only.

Correspondence concerning this article should be addressed to Mark Rubin at the School of Psychology, Behavioural Sciences Building, The University of Newcastle, Callaghan, NSW 2308, Australia. Tel: +61 (0)2 4921 6706. Fax: +61 (0)2 4921 6980. E-mail: Mark.Rubin@newcastle.edu.au Web: http://bit.ly/QgpV4O 
What happens when we die? Does the light just go out and that's that - the million-year nap? Or will some part of my personality, my me-ness, persist? What will that feel like? What will I do all day? Is there a place to plug in my laptop? Mary Roach (Roach, 2006).

I don't want to achieve immortality through my work. I want to achieve it through not dying. I don't want to live on in the hearts of my countrymen; I want to live on in my apartment. Woody Allen (Allen \& Sunshine, 1993, p. 250).

According to terror management theory (TMT; Pyszczynski, Greenberg, Solomon, \& Maxfield, 2006; Solomon, Greenberg, \& Pyszczynski, 1991), people's awareness of their own impending death causes existential anxiety, and they respond to this anxiety by adhering to cultural worldviews, which are ways of thinking about existence and reality. Cultural worldviews are assumed to reduce death-related anxiety by imbuing subjective reality with meaning, order, and stability and, hence, the possibility of symbolic immortality (e.g., being remembered by others after one has died) and/or literal immortality (e.g., continuing to exist after death in heaven).

Prior TMT research has found that experimental manipulations of thoughts of death, or mortality salience, increase the extent to which people adhere to the attitudes, values, and behaviors that are prescribed by their cultural worldviews (e.g., the greater use of stereotypes; Schimel et al., 1999). In addition, people who experience mortality salience react particularly negatively towards stimuli that threaten the meaningfulness of their cultural worldviews (e.g., Landau, Greenberg, Solomon, Pyszczynski, \& Martens, 2006; Schimel et al., 1999). However, there is currently a debate about why mortality salience causes people to adhere to and defend their cultural worldviews (for a summary, see Pyszczynski et al., 2006). The present research addressed this debate using individual difference measures of two key aspects of the fear of death: fear of self-annihilation and existential uncertainty.

\section{Fear of Self-Annihilation and Existential Uncertainty}

TMT assumes that mortality salience triggers a fear of the annihilation of the self that arises because people are concerned that the self will no longer exist after they are dead. TMT further assumes that people defend their cultural worldviews following mortality salience because cultural worldviews offer the prospective of the continuation of the self in some form after death (e.g., Landau et al., 2006, p. 888; Pyszczynski et al., 2006; Routledge \& Vess, 2012, p. 375).

However, others have challenged this view, arguing that fear of self-annihilation is not the key aspect of mortality salience that motivates worldview defense and that, instead, uncertainty surrounding death that is the more primary factor (Hohman \& Hogg, 2011; Hogg, Adelman, \& Blagg, 2010; McGregor, 2006; McGregor, Zanna, Holmes, \& Spencer, 2001; Van den Bos, Poortvliet, Maas, Miedema, \& van den Ham, 2005; Yavuz \& Van den Bos, 2009). According to this uncertainty perspective, mortality salience increases uncertainty about death, and people defend their cultural worldviews following mortality salience because doing so provides a sense of confidence in reality that helps to reduce this uncertainty. Consistent with this novel interpretation of mortality salience effects, Van den Bos et al. (2005) and Yavuz and Van den Bos (2009) found that the effects of experimental manipulations of mortality salience were limited to a subset of participants who spontaneously thought about uncertainty during the manipulations. More recently, Hohman and Hogg (2011) found that mortality salience only led to an increase in worldview defense (identifying with an ingroup) among participants who were uncertain about the 
existence of an afterlife. Finally, Martens, Burke, Schimel, and Faucher's (2011) meta-analysis showed that mortality salience is not unique in its ability to trigger worldview defense.

This past work demonstrates that mortality salience can produce uncertainty, and that uncertainty can interact with mortality salience to predict worldview defense. Critically, however, this past work does not rule out the possibility that mortality salience also evokes a fear of selfannihilation that predicts worldview defense independent of uncertainty (Van den Bos et al., 2005, p. 111). In order to properly distinguish between the self-annihilation and uncertainty perspectives, what is required is research that compares the extent to which fear of self-annihilation and fear of uncertainty about death predict worldview defense independent of one another. To address this issue, the present research assessed chronic individual differences in the fear of selfannihilation and the fear of existential uncertainty and investigated the extent to which each variable predicted cultural worldview defense independent of the other. According to TMT, fear of self-annihilation should be the primary predictor of worldview defense. In contrast, according to the uncertainty perspective, fear of uncertainty about death should be the primary predictor.

\section{The Moderating Role of the Need for Closure}

The present research also explored the need for closure as an additional predictor of worldview defense. The need for closure represents "individuals' desire for a firm answer to a question and an aversion toward ambiguity" (Kruglanski \& Webster, 1996, p. 264; Webster, \& Kruglanski, 1994). Prior research has found that the need for closure and the related construct of the need for structure interact with mortality salience to predict evaluations of stimuli that threaten the meaningfulness of cultural worldviews, such as modern art (Landau et al., 2006) and people who behave in stereotype-inconsistent ways (Schimel et al., 1999, Study 5). This prior work has shown that people with a high need for closure or structure and a salient sense of their own mortality show the most negative reactions towards stimuli that threaten the meaningfulness of their cultural worldviews. This moderating effect appears to occur because death-related thoughts reinforce the need for structure and order in cultural worldviews, and meaning threats frustrate these needs.

The need for structure has also been proposed as a moderator of the relative impact of mortality and uncertainty in predicting reactions to stimuli that threaten cultural worldviews. Commenting on Landau et al.'s (2004, Study 5) work, Van den Bos and Lind (2009) noted that people who had a high need for structure distanced themselves more from a person who threatened their worldview when mortality rather than uncertainty was salient, whereas people who had a low need for structure distanced themselves more when uncertainty rather than mortality was salient. Van den Bos and Lind concluded that the "need for structure may be an important moderator of the relative impact of mortality and uncertainty salience" (p. 129).

\section{Overview of the Present Study}

TMT assumes that thoughts of death trigger a concern about self-annihilation that motivates the defense of cultural worldviews (Landau et al., 2006; Routledge \& Vess, 2012). In contrast, uncertainty theorists propose that thoughts of death trigger a sense of uncertainty that motivates worldview defense (e.g., Hohman \& Hogg, 2011; McGregor et al., 2001; Yavuz \& Van den Bos, 2009). The present research used individual difference measures of the chronic fear of (a) self-annihilation and (b) existential uncertainty to investigate the extent to which each of these two different aspects of the fear of death predicted worldview defense. 
Following Landau et al. (2006) and Schimel et al. (1999), worldview defense was operationalised in terms of people's responses to meaning threats. Consistent with past work in this area, the need for closure was considered as an additional predictor because it has been shown to interact with mortality salience in predicting cultural worldview defense (Landau et al., 2006; Schimel et al., 1999) and to potentially moderate the differential effects of fear of self-annihilation and existential uncertainty (Van den Bos \& Lind, 2009). More specifically, people with a high need for closure and high fear of death (self-annihilation and/or uncertainty) have been shown to respond most negatively to meaning threats.

\section{Participants and Design}

\section{Method}

I recruited 463 students from first- and second-year psychology undergraduate courses at a large Australian university. Of these, 13 participants did not respond to an informed consent question at the end of the survey, and 36 indicated that they did not want their data to be included in the data analyses. I deleted the data from these 49 participants, leaving 414 participants.

The final sample consisted of 92 men and 322 women. Participants had a mean age of $22.91(S D=7.05)$. The majority of participants were White $(86.96 \%)$. I awarded course credit to 338 participants $(81.64 \%)$ in exchange for their participation. The remaining 76 participants $(18.36 \%)$ were not part of the course credit scheme.

The research used a cross-sectional experimental design, with meaning threat (meaning threat condition vs. control condition) as the between-subjects variable. Following random assignment, there were 228 participants $(55.07 \%)$ in the meaning threat condition and 186 participants $(44.93 \%)$ in the control condition.

\section{Measures}

I measured fear of self-annihilation and fear of existential uncertainty using the SelfAnnihilation and Transcendental Consequences scales of Florian and Kravetz's (1983) Personal Fear of Death scale. The four items in the Self-Annihilation scale assess fear about the destruction of the body, self, consciousness, and personality. Each item begins with the phrase "death frightens me because of..." The remaining parts of each of the four items are as follows: "the decomposition of my body," "the loss and destruction of myself," "it represents a state of everlasting sleep," and "the destruction of my personality." The four items in the Transcendental Consequences scale assess fear about uncertainty regarding what happens after death. Again, the items in this scale start with the phrase "death frightens me because of...," and they end with either "the uncertainty of what to expect," "the uncertainty of any sort of existence after death," "its mysteriousness," or "the unknowns associated with it." Participants responded to the items in these scales and the other scales in this study using a 7-point scale ranging from strongly disagree (1) to strongly agree (7). Cronbach alpha values for all scales are presented in Table 1.

I measured individual differences in the need for closure using a modified version of Webster and Kruglanski's (1994) Need for Closure scale (Roets \& Van Hiel, 2007). This scale consists of five subscales that assess the preference for predictability, closed mindedness, preference for order, discomfort with ambiguity, and decisiveness.

I manipulated meaning threat using two different photos (see below for details). I used a 4-item measure of the perceived normality of the photo as a manipulation check. The items were: "the photo did not appear strange or out of the ordinary," "the photo seemed quite normal to me," "I didn't see anything odd about the photo," and "I've seen lots of photos like this before." 
Participants evaluated the photo by responding to the following four items: "I don't rate the photo very highly," "I didn't like the photo very much," "the photo is quite nice," and "I would hang the photo on my wall." I reverse-scored the last two items. Hence, this measure assessed the extent to which participants disliked the photo.

I used a measure of general anxiety to determine whether any observed effects could be explained in relation to anxiety rather than death-related fear. I assessed anxiety using the 21-item State-Trait Inventory for Cognitive and Somatic Anxiety scale (Ree, French, MacLeod, \& Locke, 2008). The trait version of this scale asked participants to respond according to how they felt generally for most of the time, and the state version asked participants to respond as they felt right now at this precise moment in time.

Finally, I used Rubin, Paolini, and Crisp's (2010) 4-item Perceived Awareness of the Research Hypothesis scale to measure the potential influence of demand characteristics on the observed effects. This scale measures the extent to which participants believe that they are aware of the research hypotheses (for a discussion, see Rubin, 2016). An example item is "I knew what the researchers were investigating in this research." If demand characteristics influenced the observed effects, then scores on this scale should be significantly related to those effects. ${ }^{1}$

\section{Experimental Manipulation of Meaning Threat}

Past research has effectively manipulated meaning threat by exposing different groups of participants to specific paintings that vary in their level of meaningfulness (e.g., Proulx et al., 2010, Study 3; Routledge, Wildschut, Sedikides, Juhl, \& Arndt, 2012, Experiment 3). I used a similar approach in the present research. In the meaning threat condition, participants viewed a photo of a fisherman standing on the deck of a fishing boat. Inexplicably, the fisherman's head has been replaced with a horse's head, and he is holding a cat. In the control condition, participants viewed a similar photo of a fisherman working on the deck of the same boat with his back turned to the camera. There was nothing out of the ordinary in this second photo (i.e., no horse's head and no cat). The two photos are shown in Figure 1.
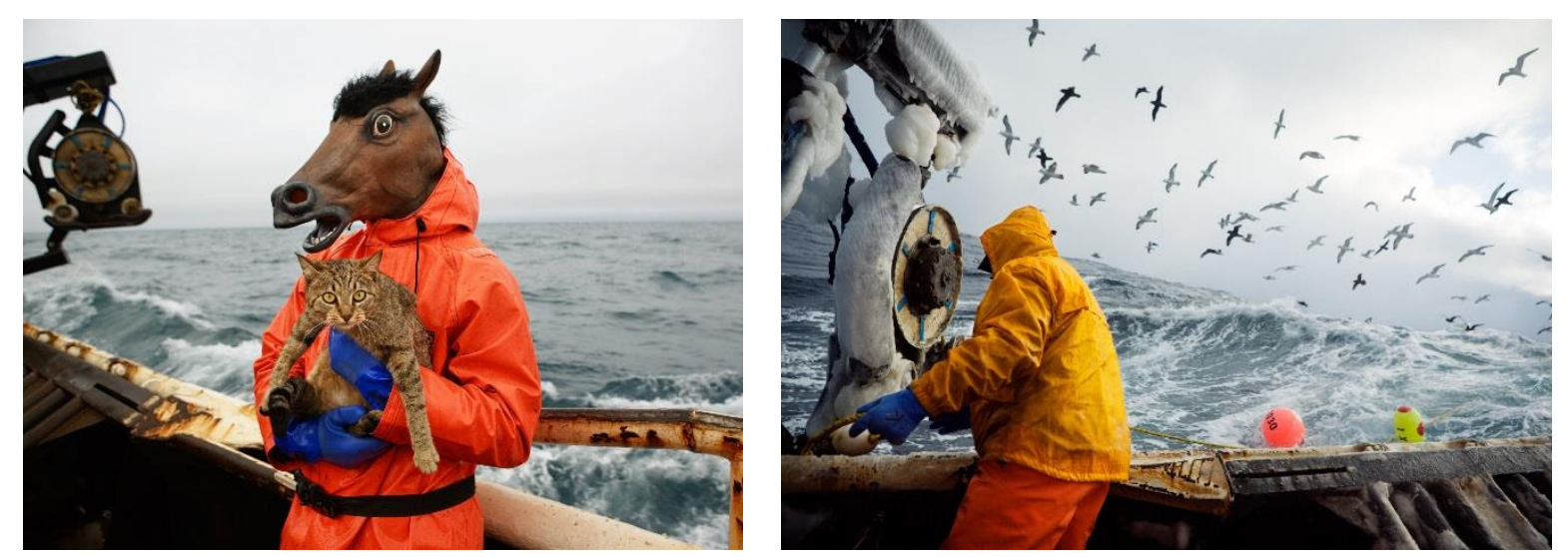

Figure 1. The photos displayed to participants in the meaning threat condition (left photo) and control condition (right photo). () Corey Arnold. I am grateful to Corey Arnold for granting permission to reproduce these photos.

The survey instructions asked participants to study the photo very carefully for at least one minute. Participants read that they would be asked to explain their understanding of the photo at a later point during the survey. 


\section{Procedure}

The survey introduced the research as "investigating how certain personality variables predict people's evaluations of a particular photograph." Participants completed the anonymous online survey in their own time. The survey took 20-30 minutes to complete.

I presented the measures of need for closure, fear of self-annihilation, fear of existential uncertainty, and trait anxiety prior to the experimental manipulation of meaning threat. I measured trait anxiety first followed by the need for closure and fear of death measures, which I presented in a random order. I presented the measures of state anxiety, perceived normality of the photo, photo evaluation, and perceived awareness of the research hypothesis after the experimental manipulation and in that order, with the items from the second and third measures presented together but in a randomised order. I randomised the order of presentation of items from the same measures for each participant.

\section{Type I Error Control}

\section{Results}

The multiple testing that I undertook in the analyses for this study increased the probability of Type I (false positive) errors. To control for this increased error rate, I lowered the alpha level for determining significant results. The revised alpha level was based on Benjamini and Hochberg's (1995) procedure to control the false discovery rate (FDR). ${ }^{2}$ The FDR is based on the frequency of true null hypotheses in a specified data set as indicated by the distribution of $p$ values in that data set. To estimate the frequency of true null hypotheses in the current data set, I performed a zero-order correlation analysis that included all of the measures in the survey apart from categorical variables with more than two levels, which were inappropriate for a correlation analysis. For psychometric scales, only overall scales were included rather subscales or individual scale items. This approach prevented the artificial inflation of significant associations. The correlation analysis yielded a total of $460 p$ values. I then used an FDR of 5\% to identify the critical $p$ value at which nonsignificant results became significant. This value was .034 , and it was used as the alpha level in my subsequent analyses. Using this adjusted alpha level, a maximum of 1 out of every 20 significant results represent Type I errors.

\section{Manipulation Check}

I used an independent samples $t$ test to investigate the effect of the experimental manipulation of meaning threat on the perceived normality of the photo. There was a significant effect of the manipulation, $t(412)=15.31, p<.001,95 \%$ CI $(1.52,1.97)$. Participants in the meaning threat (horse's head) condition rated the photo as being less normal $(M=3.02, S D=1.23)$ than participants in the control condition $(M=4.77, S D=1.06)$.

\section{Main Analyses ${ }^{3}$}

Means, standard deviations, and zero-order correlations among continuous variables are presented in Table 1.

I conducted a multiple regression analysis that included fear of existential uncertainty, fear of self-annihilation, the need for closure, the meaning threat manipulation, and the corresponding two-, three-, and four-way interaction terms as predictor variables. Evaluation of the photo was the outcome variable. The meaning threat manipulation was contrast coded as -1 for control and 1 for meaning threat. I centred all variables by converting to $z$ scores prior to creating interaction 
Table 1

Descriptive Statistics and Zero Order Correlation Coefficients

\begin{tabular}{|c|c|c|c|c|c|c|c|c|c|c|}
\hline Measure & $M$ & $S D$ & $\mathrm{~A}$ & 1 & 2 & 3 & 4 & 5 & 6 & 7 \\
\hline Self-annihilation & 3.26 & 1.65 & .87 & - & & & & & & \\
\hline Existential uncertainty & 3.99 & 1.94 & .94 & $.74 * *$ & - & & & & & \\
\hline Need for closure & 4.46 & 0.60 & .88 & $.20 * *$ & $.23 * *$ & - & & & & \\
\hline $\begin{array}{l}\text { Perceived normality of } \\
\text { photo }\end{array}$ & 3.81 & 1.44 & .84 & $-.13 * *$ & $-.01 *$ & -.01 & - & & & \\
\hline Photo evaluation & 4.29 & 1.34 & .85 & .10 & $.11 *$ & $.14 * *$ & $-.42 * *$ & - & & \\
\hline Trait anxiety & 3.55 & 1.10 & .93 & $.14 * *$ & $.16^{* *}$ & $.36^{* *}$ & -.02 & .10 & - & \\
\hline State anxiety & 3.19 & 1.13 & .93 & $.19 * *$ & $.20 * *$ & $.32 * *$ & -.01 & $.11 *$ & $.83^{* *}$ & - \\
\hline PARH & 3.74 & 1.31 & .91 & .05 & .07 & -.07 & .01 & $-.11 *$ & .00 & .02 \\
\hline
\end{tabular}

Note. All $n \mathrm{~s}=414$. PARH $=$ Perceived awareness of the research hypothesis

$* p<.034$ (adjusted alpha). $* * p<.01$.

terms. Note that common method variance was likely to be relatively high between the fear of self-annihilation and fear of existential uncertainty predictors because the items in these two scales began with the same phrase ("death frightens me because of...") and they both relate to the same subject matter (fear of death). However, concerns about multicollinearity were allayed by the fact that all variance inflation factors for this multiple regression analysis were $\leq 3.04$.

The multiple regression analysis found significant overall positive effects for the need for closure, $B=0.41, S E=0.14, t(398)=2.85, p=.005,95 \% \mathrm{CI}(0.13,0.69)$, and the meaning threat manipulation, $B=0.36, S E=0.08, t(398)=4.41, p<.001,95 \% \mathrm{CI}(0.20,0.52)$. These overall effects were qualified by a significant two-way interaction between the need for closure and the meaning threat manipulation, $B=0.19, S E=0.09, t(398)=2.24, p=.026,95 \%$ CI $(0.23,0.36)$, which was itself qualified by a significant three-way interaction between fear of self-annihilation, the need for closure, and the meaning threat manipulation, $B=0.25, S E=0.10, t(398)=2.55, p=$ $.011,95 \% \mathrm{CI}(0.06,0.44)$. No other effects were significant $(p \mathrm{~s} \geq .120)$, including the three-way interaction between fear of existential uncertainty, the need for closure, and the meaning threat manipulation, $B=-0.07, S E=0.10, t(398)=-0.70, p=.485,95 \% \mathrm{CI}(-0.27,0.13)$.

It is difficult to reach firm conclusions about the null effect of fear of existential uncertainty using null hypothesis significance testing. To arrive at firmer conclusions, I conducted a Bayesian regression analysis that compared the two critical three-way interaction effects on photo evaluation. In other words, I contrasted the three-way interaction between fear of existential uncertainty, the need for closure, and the manipulation of meaning threat with the three-way interaction between fear of self-annihilation, the need for closure, and the manipulation of meaning threat. The analysis was conducted using the R BayesFactor package, and the prior scale (Cauchy distribution) was set to medium, based on the assumption that effect sizes would be small. The Bayes Factor for this comparison was 13.69 in favor of the self-annihilation interaction, indicating that this interaction effect was 13.69 times more likely to predict photo evaluation than the interaction involving existential uncertainty. Hence, contrary to the uncertainty theory predictions, this Bayesian analysis provides "strong" evidence (Jeffreys, 1961) for the hypothesis that the interaction involving fear of self-annihilation predicts worldview defense better than the interaction involving fear of existential uncertainty.

I used Hayes' (2013) PROCESS software to further investigate the significant three-way interaction between fear of self-annihilation, the need for closure, and the meaning threat manipulation. This test investigated fear of self-annihilation and the need for closure as moderators of the effect of the meaning threat manipulation on photo evaluation while controlling for fear of existential uncertainty. The pattern of significant and nonsignificant results remained the same when fear of existential uncertainty was not included as a covariate in this analysis. 
Replicating the effects observed in the initial analysis, there were significant overall effects of the need for closure, $B=0.31, S E=0.11, t(405)=2.84, p=.005,95 \% \mathrm{CI}(0.10,0.53)$, and the meaning threat manipulation, $B=0.32, S E=0.06, t(405)=5.11, p<.001,95 \% \mathrm{CI}(0.20,0.45)$. These effects were qualified by a two-way interaction between the need for closure and the meaning threat manipulation, $B=0.32, S E=0.11, t(405)=2.94, p=.004,95 \% \mathrm{CI}(0.11,0.53)$, and a three-way interaction between fear of self-annihilation, the need for closure, and the meaning threat manipulation, $B=0.18, S E=0.06, t(405)=2.81, p=.005,95 \%$ CI $(0.05,0.30)$. To investigate the three-way interaction, I tested the two-way interaction between the need for closure and the meaning threat manipulation at low, medium, and high levels of fear of self-annihilation. Following Hayes (2013), low levels were defined as being at one standard deviation below the mean, medium levels were defined as being at the mean value, and high levels were defined as being at one standard deviation above the mean. The two-way interaction between the need for closure and the meaning threat manipulation was nonsignificant at low levels of the fear of selfannihilation, $B=0.03, S E=0.13, t(405)=0.21, p=.836,95 \% \mathrm{CI}(-0.23,0.29)$, stronger and significant at medium levels, $B=0.32, S E=0.11, t(405)=2.94, p=.004,95 \%$ CI $(0.11,0.53)$, and strongest and significant at high levels, $B=0.61, S E=0.17, t(405)=3.68, p<.001,95 \% \mathrm{CI}$ $(0.29,0.94)$. An analysis of the effect of the meaning threat manipulation as a function of both fear of self-annihilation and the need for closure found that, at all levels of fear of self-annihilation, the effect of the meaning threat manipulation on photo evaluation became progressively stronger moving from small through medium to high levels of the need for closure. However, this trend became more pronounced with increasing levels of fear of self-annihilation.

To illustrate these results, I subtracted mean levels of photo evaluation in the control condition from mean levels in the meaning threat condition. Positive values on the resulting index indicated that participants in the meaning threat condition disliked the photo more than participants in the control condition. Figure 2 shows the size of this relative dislike at each level of fear of selfannihilation and the need for closure. As can be seen in Figure 2, relative dislike of the meaning threat photo increased with increasing need for closure, and this trend became more pronounced with increasing levels of fear of self-annihilation.

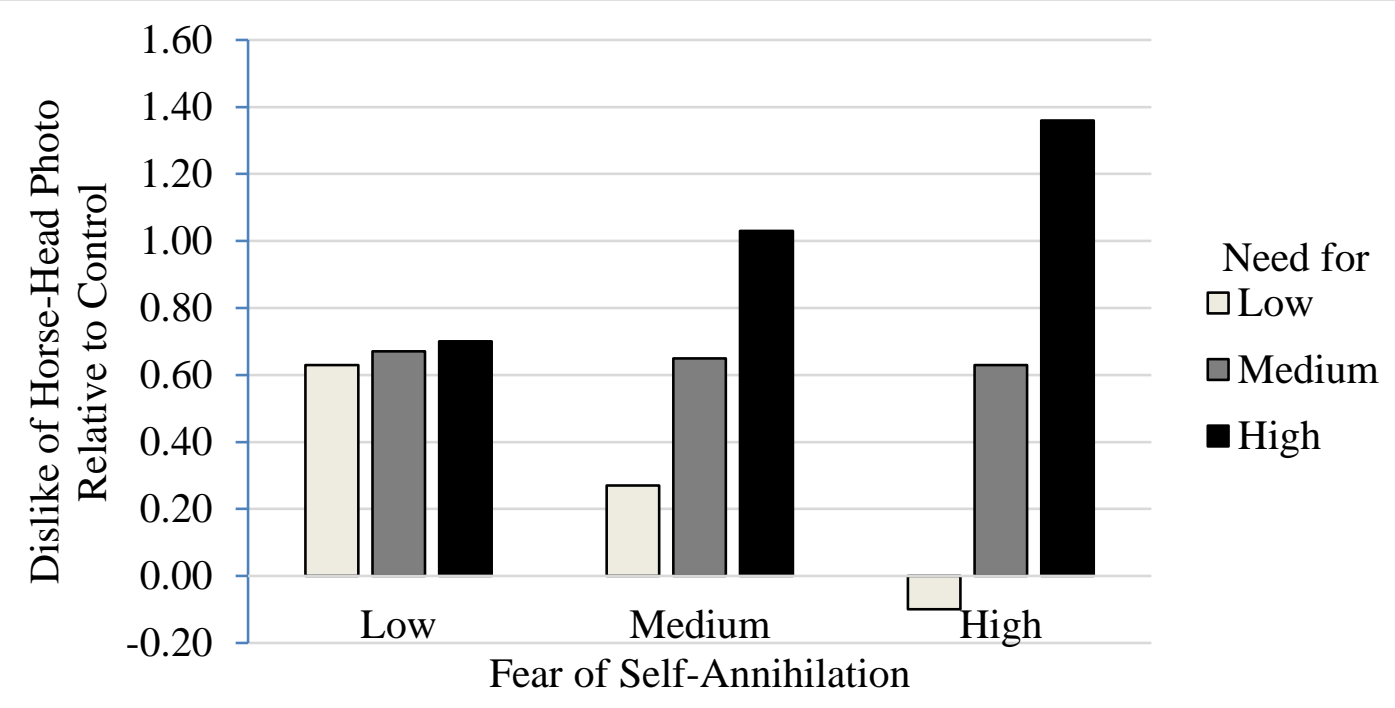

Figure 2. Dislike for the meaning threat photo relative to the control photo as a function of fear of self-annihilation and the need for closure. Note that values represent difference scores (meaning threat condition minus control condition) in which more positive values indicate that participants in the meaning threat condition disliked the photo more than those in the control condition. 
I also considered Van den Bos and Lind's (2009) proposal that the need for structure moderates the relative impact of mortality and uncertainty. In the context of the present study, this proposal can be interpreted as predicting that people who have a high need for closure should dislike the meaning threat photo more when they have a high rather than a low fear of selfannihilation, and people who have a low need for closure should dislike the meaning threat photo more when they have a high rather than low fear of existential uncertainty. The results show that, consistent with TMT, the first of these predictions was borne out: People who had a high need for closure and a high fear of self-annihilation reported the greatest dislike of the meaning threat photo relative to the control condition. However, contrary to Van den Bos and Lind's predictions, people who had a low need for closure and a high fear of existential uncertainty showed the lowest levels of dislike of the meaning threat photo relative to the control condition, not the highest. Hence, rather than moderating the extent to which fear of self-annihilation and existential uncertainty are related to evaluations of meaning threats, the moderating role of the need for closure was restricted to fear of self-annihilation.

\section{Discussion}

TMT theorists propose that thoughts of death trigger a concern about self-annihilation that motivates the defense of cultural worldviews (Landau et al., 2006; Routledge \& Vess, 2012). In contrast, uncertainty theorists propose that thoughts of death trigger a sense of uncertainty that motivates worldview defense (e.g., Hohman \& Hogg, 2011; McGregor et al., 2001; Yavuz \& Van den Bos, 2009). The present research used individual difference measures of the fear of selfannihilation and existential uncertainty to explore the extent to which each of these two aspects of the fear of death predicted negative reactions to a meaning threat.

Consistent with prior research (Rubin, Paolini, \& Crisp, 2011), individual differences in the need for closure predicted the extent to which people disliked a meaning threat stimulus (a photo of a fisherman with a horse's head and holding a cat) relative to a control stimulus (a photo of a fisherman with a normal head and no cat). The greater people's need for closure, the more they disliked the photo in the meaning threat condition relative to the control condition.

Consistent with TMT, this effect was moderated by chronic individual differences in the fear of self-annihilation. People who had a strong fear about the destruction of their self after death showed the strongest positive relationship between the need for closure and dislike of the meaning threat photo. Indeed, people who had a high fear about self-annihilation and a high need for closure reported the strongest dislike of the meaning threat photo. From a TMT perspective, this pattern of results occurred because the meaning threat photo challenges the usefulness of people's cultural worldviews, which are unlikely to explain why a fisherman on a fishing boat has a horse's head and is holding a cat. Cultural worldviews provide the potential for symbolic and/or literal selfpreservation following death to the extent that they provide a sense of order, stability, and predictability. Hence, people who have a chronic fear of death-related self-annihilation and a strong need for order, stability, and predictability (i.e., a high need for closure) are most likely to defend their cultural worldviews by derogating (disliking) stimuli that threaten the perceived usefulness of these worldviews.

It is interesting to note that the pattern of results illustrated in Figure 2 is due to both increasing dislike when the need for closure is high and decreasing dislike when need for closure is low. In other words, as fear of self-annihilation increased, people who had a high need for closure showed greater dislike of the meaning threat photo, and people who had a low need for closure showed less dislike of this photo. Indeed, when fear of self-annihilation was medium or 
high, people who had a low need for closure showed no significant difference in their evaluation of the meaning threat photo compared to people who rated the control photo ( $p s \geq .149)$. This pattern of result is consistent with TMT's assumptions. People who have a low need for closure value unpredictability, open mindedness, creativity, and ambiguity and, somewhat ironically, a high fear of self-annihilation should motivate them to adhere more strongly to these values because they form an important part of their cultural worldview. Hence, fear of self-annihilation should lead people who have a low need for closure to be more tolerant of unpredictable stimuli (for similar arguments, see Landau et al., 2006, p. 887; Schimel et al., 1999, p. 920).

The present results are similar to those obtained in previous studies that have investigated mortality salience and the need for closure or structure as predictors of evaluations of meaning threats (Landau et al., 2006; Schimel et al., 1999, Study 5). However, the current research extends on this prior work by explicitly contrasting fear of self-annihilation with fear of existential uncertainty. Contrary to the uncertainty perspective, fear of existential uncertainty did not operate as a significant predictor of worldview defense in the present research, either independently or in interaction with other predictors. Furthermore, the need for closure did not moderate the extent to which fear of existential uncertainty predicted worldview defense (Van den Bos \& Lind, 2009). Hence, the present evidence did not support the uncertainty perspective. Instead, it provides more support for TMT because it suggests that fear of self-annihilation predicts worldview defense independent of uncertainty (e.g., Pyszczynski et al., 2006; Routledge \& Vess, 2012).

To be clear, the current evidence does not imply that only the fear of self-annihilation predicts worldview defense (see also Pyszczynski et al., 2006, p. 331). Prior research has demonstrated that, under some circumstances, death-related uncertainty is also related to worldview defense (e.g., Hohman \& Hogg, 2011; McGregor, 2006; Van den Bos et al., 2005). However, consistent with TMT, the current evidence does demonstrate that, under some circumstances, fear of self-annihilation predicts worldview defense independent from fear of existential uncertainty, and this finding throws doubt on the suggestion that death-related effects are driven by a fear of existential uncertainty (for a discussion, see Pyszczynski et al., 2006). In this sense, the present research results converge with Martens et al.'s (2011) conclusion that deathrelated thoughts have a "distinct and unique" impact on worldview defense (p. 10).

A key limitation of the methodology used in the present research is that the Transcendental Consequences subscale only measured uncertainty about existence after death (Landau et al., 2006). It did not measure other forms of uncertainty surrounding death, such as when it will happen and what it will feel like (Hohman \& Hogg, 2011; Van der Bos \& Lind, 2009). Hence, although the present research suggests that fear of self-annihilation is more clearly associated with worldview defense than fear of existential uncertainty, it does not indicate whether other forms of death-related uncertainty play an important role. Future research should explore this issue by differentiating between different aspects of death-related uncertainty.

It is also important to note that the present research conclusions are based on a single study. Future research should attempt to replicate the current research findings, perhaps in relation to an alternative type of worldview defense (e.g., stereotyping). One approach would be to both experimentally manipulate and measure fear of self-annihilation and fear of existential uncertainty and observe their effects on worldview defense. This research design would allow researchers to investigate the causal effects of each type of fear on worldview defense while controlling for the measured effects of the other type of fear. 
In summary, the present study finds tentative support in favour of TMT's proposal that fear of self-annihilation predicts worldview defense independent of uncertainty. However, further research is required in order to confirm the validity of this finding.

\section{References}

Allen, W., \& Sunshine, L. (1993). The illustrated Woody Allen reader. New York: Knopf.

Benjamini, Y., \& Hochberg, Y. (1995). Controlling the false discovery rate: A practical and powerful approach to multiple testing. Journal of the Royal Statistical Society. Series B (Methodological), 57, 289-300.

Florian, V., \& Kravetz, S. (1983). Fear of personal death: Attribution, structure, and relation to religious belief. Journal of Personality and Social Psychology, 44, 600-607. doi: 10.1037/0022-3514.44.3.600

Glickman, M. E., Rao, S. R., \& Schultz, M. R. (2014). False discovery rate control is a recommended alternative to Bonferroni-type adjustments in health studies. Journal of Clinical Epidemiology, 67, 850-857. doi: 10.1016/j.jclinepi.2014.03.012

Hayes, A. F. (2013). An introduction to mediation, moderation, and conditional process analysis: A regression-based approach. New York: Guilford Press.

Hogg, M. A., Adelman, J. R., \& Blagg, R. D. (2010). Religion in the face of uncertainty: An uncertainty-identity theory account of religiousness. Personality and Social Psychology Review, 14, 72-83. doi: 10.1177/1088868309349692

Hohman, Z. P., \& Hogg, M. A. (2011). Fear and uncertainty in the face of death: The role of life after death in group identification. European Journal of Social Psychology, 41, 751-760. doi: 10.1002/ejsp.818

Kruglanski, A. W., \& Webster, D. M. (1996). Motivated closing of the mind: "Seizing" and "freezing." Psychological Review, 103, 263-283. doi: 10.1037/0033-295X.103.2.263

Jeffreys, H. (1961). The theory of probability (3rd ed.). Oxford, UK: Oxford University Press.

Landau, M. J., Greenberg, J., Solomon, S., Pyszczynski, T., \& Martens, A. (2006). Windows into nothingness: Terror management, meaninglessness, and negative reactions to modern art. Journal of Personality and Social Psychology, 90, 879-892. doi: 10.1037/00223514.90.6.879

Landau, M. J., Johns, M., Greenberg, J., Pyszczynski, T., Martens, A., Goldenberg, J. L., \& Solomon, S. (2004). A function of form: Terror management and structuring the social world. Journal of Personality and Social Psychology, 87, 190-210. doi: 10.1037/00223514.87.2.190

Martens, A., Burke, B. L., Schimel, J., \& Faucher, E. H. (2011). Same but different: Metaanalytically examining the uniqueness of mortality salience effects. European Journal of Social Psychology, 41, 6-10. doi: 10.1002/ejsp.767

McGregor, I. (2006). Offensive defensiveness: Toward an integrative neuroscience of compensatory zeal after mortality salience, personal uncertainty, and other poignant selfthreats. Psychological Inquiry, 17, 299-308. doi: 10.1080/10478400701366977

McGregor, I., Zanna, M. P., Holmes, J. G., \& Spencer, S. J. (2001). Compensatory conviction in the face of personal uncertainty: Going to extremes and being oneself. Journal of Personality and Social Psychology, 80, 472-488. doi: 10.1037/0022-3514.80.3.472

Osborne, J. W., \& Overbay, A. (2004). The power of outliers (and why researchers should always check for them). Practical Assessment, Research \& Evaluation, 9, 1-12. 
Proulx, T., Heine, S. J., \& Vohs, K. D. (2010). When is the unfamiliar the uncanny? Meaning affirmation after exposure to absurdist literature, humor, and art. Personality and Social Psychology Bulletin, 36, 817-829. doi: 10.1177/0146167210369896

Pyszczynski, T., Greenberg, J., Solomon, S., \& Maxfield, M. (2006). On the unique psychological import of the human awareness of mortality: Theme and variations. Psychological Inquiry, 17, 328-356. doi: 10.1080/10478400701369542

Ree, M. J., French, D., MacLeod, C., \& Locke, V. (2008). Distinguishing cognitive and somatic dimensions of state and trait anxiety: Development and validation of the State-Trait Inventory for Cognitive and Somatic Anxiety (STICSA). Behavioural and Cognitive Psychotherapy, 36, 313-332. doi: 10.1017/S1352465808004232

Roach, M. (2006). Spook: Science tackles the afterlife. New York: Norton.

Roets, A., \& Van Hiel, A. (2007). Separating ability from need: Clarifying the dimensional structure of the Need for Closure scale. Personality and Social Psychology Bulletin, 33, 266-280. doi: 10.1177/0146167206294744

Routledge, C., \& Vess, M. (2012). More than meets the eye: There's more to meaning maintenance than violated expectations. Psychological Inquiry, 23, 374-380. doi: 10.1080/1047840X.2012.721078

Routledge, C., Wildschut, T., Sedikides, C., Juhl, J., \& Arndt, J. (2012). The power of the past: Nostalgia as a meaning-making resource. Memory, 20, 452-460. doi: 10.1080/09658211.2012.677452

Rubin, M., Paolini, S., \& Crisp, R. J. (2010). A processing fluency explanation of bias against migrants. Journal of Experimental Social Psychology, 46, 21-28. doi: 10.1016/j.jesp.2009.09.006

Rubin, M., Paolini, S., \& Crisp, R. J. (2011). The relationship between the need for closure and deviant bias: An investigation of generality and process. International Journal of Psychology, 46, 206-213. doi: 10.1080/00207594.2010.537660

Rubin, M. (2016). The Perceived Awareness of the Research Hypothesis Scale: Assessing the influence of demand characteristics. Figshare. doi: 10.6084/m9.figshare.4315778

Schimel, J., Simon, L., Greenberg, J., Pyszczynski, T., Solomon, S., Waxmonsky, J., \& Arndt, J. (1999). Stereotypes and terror management: Evidence that mortality salience enhances stereotypic thinking and preferences. Journal of Personality and Social Psychology, 77, 905-926. doi: 10.1037/0022-3514.77.5.905

Solomon, S., Greenberg, J., \& Pyszczynski, T. (1991). A terror management theory of social behavior: The psychological functions of self-esteem and cultural worldviews. Advances in Experimental Social Psychology, 24, 91-159.

Van den Bos, K., \& Lind, E. A. (2009). The social psychology of fairness and the regulation of personal uncertainty. In R. M. Arkin, K. C. Oleson, \& P. J. Carroll (Eds.), Handbook of the uncertain self (pp. 122-141). New York: Psychology Press.

Van den Bos, K., Poortvliet, P. M., Maas, M., Miedema, J., \& van den Ham, E. J. (2005). An enquiry concerning the principles of cultural norms and values: The impact of uncertainty and mortality salience on reactions to violations and bolstering of cultural worldviews. Journal of Experimental Social Psychology, 41, 91-113. doi: 10.1016/j.jesp.2004.06.001

Webster, D. M., \& Kruglanski, A. W. (1994). Individual differences in need for cognitive closure. Journal of Personality and Social Psychology, 67, 1049-1062. doi: 10.1037/00223514.67.6.1049 
Yavuz, H., \& Van den Bos, K. (2009). Effects of uncertainty and mortality salience on worldview defense reactions in Turkey. Social Justice Research, 22, 384-398. doi: 10.1007/s11211009-0104-5

\section{Endnotes}

1. The survey also included self-report measures of creativity, self-esteem, sensation-seeking, the need for cognition, processing fluency, meaning compensation strategies, and ability to understand multiple meanings. These measures did not yield any results that related to current research questions, and so they are not discussed further. A copy of the research survey and data is available at https://osf.io/e8krj/

2. The FDR is the maximum proportion of Type I errors ("false discoveries") that can be expected for a particular data set. The FDR adjustment is more powerful than the Bonferroni adjustment because it limits the proportion of false positive (Type I) results to a certain percentage (e.g., $5 \%$ ) rather than reduces the chances of making a single false positive error. To illustrate, imagine that a researcher conducts 100 independent tests with an alpha level of .05 . In this case, the researcher will have a $99.41 \%$ probability of obtaining at least one Type I error (Glickman, Rao, \& Schultz, 2014). However, with an FDR of .05, the proportion of Type I errors can be controlled to a maximum of $5 \%$ of the significant results without sacrificing sufficient power to detect genuine effects (Glickman et al., 2014). The FDR adjustment is based on the frequency of true null hypotheses in a specified data as indicated by the distribution of $p$ values in that data set (Glickman et al., 2014). Hence, data sets that contain a higher frequency of true null hypotheses (as indicated by a less skewed distribution of $p$ values) need to have a lower alpha level in order to achieve a 5\% FDR.

3. Continuous variables were checked for outliers using a +/- 3 SDs criterion (Osborne \& Overbay, 2004). There was one outlier on the need for closure variable. The pattern of significant and nonsignificant results that are reported remained the same when this outlier was excluded from the analyses. The analyses were also repeated including (a) trait anxiety and perceived awareness of the research hypothesis as covariates and (b) state anxiety and perceived awareness of the research hypothesis as covariates. Again, the pattern of significant and nonsignificant results remained the same with these covariates included in the analyses. Hence, the effects reported above do not appear to be related to either general trait anxiety, general state anxiety, or demand characteristics.

Funding

The author declares no funding sources.

\section{Conflict of Interest}

The author declares no conflict of interest. 